\title{
Provisioning of Optimized Cab Services in Secured Blockchain Framework
}

\author{
Manoj B. Chandak \\ Department of Computer Science and Engineering Shri Ramdeobaba \\ College of Engineering and Management, Nagpur, India
}

\section{ABSTRACT}

Modern online cab booking services such as Ola and Uber are becoming increasingly popular due to ease of use and cost effectiveness. With rising popularity comes the responsibility of providing quality service to the customers. The presented research work illustrates a system that aims to provide maximum customer satisfaction by reducing the waiting time for a cab, improvising on the availability of cabs and providing data security. Availability of the cabs can be improved by reducing the distance between the locations of available cabs and the customers. One of the methods to reduce the distance is the prediction of cab bookings and ensuring availability of the cab service to the potential customer location in advance. The presented paper proposes a prediction mechanism based on a data set of social events happening in a particular region. As social events normally bring masses of participants at a location, the probability of use of cab services increases at that location. A Machine Learning (ML) model is built to predict the number of cab bookings for different areas, based on historical data \&t the type of participants attending the event. The predicted number of required cabs, current cab locations and event location are then used by the designed system to find the nearest drivers from the event location. The nearest drivers are notified to reach the location beforehand thereby making cabs readily available when the requirement arises. To ensure security of data in the compact system, private Blockchain network is used for data transfer and storage, making it reliable while also maintaining data integrity and confidentiality..

KEY WORDS: BLOCKCHAIN, ETHEREUM, SMART CONTRACT, MACHINE LEARNING, ARTIFICIAL NEURAL NETWORK, K-NEAREST NEIGHBOUR.

\section{INTRODUCTION}

Concept of organized rental cab was introduced to the Indian consumer market in 2004. It was first introduced by Meru cab service in major metro cities of India. Almost, after a gap of six years, app based rental cab services were introduced to the Indian consumers. Amongst various transportation modes, cab services gained popularity as

\section{ARTICLE INFORMATION}

Received 9th Oct 2020 Accepted after revision 25th Dec 2020 Print ISSN: 0974-6455 Online ISSN: 2321-4007 CODEN: BBRCBA

Thomson Reuters ISI Web of Science Clarivate Analytics USA and Crossref Indexed Journal

\section{Clarivate
Analytics}

NAAS Journal Score 2020 (4.31) SJIF: 2020 (7.728)

A Society of Science and Nature Publication,

Bhopal India 2020. All rights reserved.

Online Contents Available at: http//www.bbrc.in/

Doi: http://dx.doi.org/10.21786/bbrc/13.14/62 they facilitate door to door service for consumers. Due to advancement in technology, operational procedures like bookings and payments got automated, which increased the overall potential of the business domain. As the popularity increased, the scarcity and non-availability of cab at desired locations also increased which resulted in escalated waiting time for the customers. The escalation was largely observed during peak business hours. After the launch of Uber services in 2013 [Rajesh (2018)], competition in the business domain intensified. Eventually the service providers started creating better and simpler automated services for the customers.

As customers became more demanding, it became a challenging job for rental cab industry to live up to the expectations. In today's world not only the price but also the quality of cab services plays an important role

\section{4}


in customer satisfaction. The presented research work describes the system design, implementation and test cases to improve the quality of cab services. Hence it aims at providing maximum customer satisfaction with the help of modern technologies such as Artificial Intelligence, Machine Learning (ML) and Blockchain.

Proposed Methodology: Machine Learning for Prediction: Online cab booking services are extensively used in the modern world. The services are easily accessible anytime, anywhere by the use of a simple mobile application. After a customer books a cab, he/she has to wait for the cab to arrive. The delay in arrival depends on the distance between the customer and the cab at the time of booking. Reducing the distance reduces the waiting time and therefore improves quality of cab service. One of the ways to reduce is by the use of prediction systems. To predict the number of cab bookings from a location, prediction system makes use of historical data. The concerned cab drivers are notified beforehand which dramatically reduces and consequently reduces waiting time.

The quality of data used for prediction is of utmost importance. Therefore various cab booking patterns were observed as datasets to accurately predict the bookings. It was found that booking hotspots may form at certain locations because of events like arrival of a train at a station or some social gathering. Various such event data can be used for building the prediction system. The proposed system uses information about happenings and events in a city or locality to predict the customer cab booking at a location. Publicly available event data from a website (meetup.com) is used as a data source for the operation of system. Prediction is feasible as the number of cab bookings at a location is dependent on the number of people attending social events and gatherings near the same location.

In other words,

\section{$E \propto B$}

Where, Eis the number of people attending an event and is the number of cab bookings in that area.

The probable sequence of actions that a customer may take before reaching for a cab is as follows:

- Customer looks for a social event on a website and registers for it.

- On the day of the event, the customer decides to take a cab to reach the location of the event.

- After the event, the customer takes a cab to reach home/some other destination.

The presented research work mainly focuses on the 3rdaction. Data from an event portal is used to predict customers who decide to take cab from the event location. The Prediction system makes use of ML. A trained ML model with satisfactory accuracy is required for deployment. Whenever a new event appears on the event portal, the ML model must start predicting cab bookings without delay. The prediction provides an estimation of bookings from the spot around the event location. The estimation is used to send the available cab drivers to the event location in advance. For the drivers to arrive early, they are notified about the estimation around the time the event takes place. In a standard cab system, only nearby cabs are able to reach the destination in reasonable time thus only the nearest drivers are notified. K-Nearest Neighbours (KNN) algorithm is used to determine the nearest available cabs, given the required number $\mathrm{K}$.

\section{Implementation}

Prediction Model: The process of building an ML model prediction system involves data selection, data extraction, data pre-processing, selecting an ML model and, finally training, evaluating and tuning a model. The first step towards building an ML model is selecting an appropriate dataset. The dataset used in the system consists of information about social events and gatherings in a city or locality. Website portals like meetup.com where users register for local events, provide a public event dataset, making them a good data source. The system makes use of public dataset provided by meetup.com which consists of various attributes and information about events. Among the various attributes available, only a few are selected based on their relationship with the required, prediction of number of cab bookings.

- Event Type like Social Gathering, Technical Workshop, Volunteering, etc (Cab bookings can depends on the type of people attending the event).

- Event Organiser ID (An organiser's reputation has an effect on the number of people attending)

- Number of Registrations (Is directly related to number of people attending)

- Positive and Negative Comments (Reputation of event has an effect on the number of people attending)

- $\quad$ Rating of Event

To process the public dataset further, following Python Programming Language libraries are used:

1) NumPy 2) Pandas. To build the ANN model, Keras library was used.

Blockchain: The implementation of the blockchain system involves configuring a private blockchain, writing scripts for data transfer and finally, compiling and deploying the scripts on blockchain network. For the configuration of the private blockchain network, Ganache software is used. Ganache is an in-memory virtual Ethereum blockchain network for developing and testing blockchain Applications locally. It is configured to create some virtual blockchain accounts (Blockchain IDs) and a port is specified to listen for blockchain requests. Each virtual account consists of a Public-Private Key pair.

After configuring Ganache, the next step is to write a script that enables data transfer via blockchain network i.e a Smart Contract. A Smart Contract is a self-executing Contract with a set of defined tasks and functions to 
perform. The code contained in a Smart Contract exists across the nodes in distributed blockchain network after its deployment. The code controls process execution and makes the transactions trackable and irreversible. The Smart Contract is written in a high level Object Oriented Language called Solidity. It is then compiled into bytecode and deployed on the blockchain network.

In the system presented, a Solidity file "CabService.sol" is used. The structure of the solidity file includes CabService as "contract". In the "CabService.sol" file, the function "sendLocation" is used to interact with blockchain. The main task of the "sendLocation" function is to transfer location data to the blockchain network. The function has id and location (as latitude and longitude) of a driver as the input parameters. To compile and deploy contracts on the blockchain, Truffle framework is used.

Implementation of Android Application: An accompanying Mobile Application for the cab drivers is an integral part of the proposed system. The main purpose of the Application is to transfer the location of drivers from their mobile devices to the blockchain network securely. The application has to establish connection with the blockchain network while hiding its details from the user. Focus on responsive User Interface (UI) is also necessary for good user experience. An Android application was developed for the purpose of testing the prototype system. There are 2 major steps for implementing the Android Application i.e. connection configuration and data transfer to blockchain network.

Connection configuration requires setting up IP addresses and port numbers along with the Smart Contract address, Public-Private key pair and Solidity file in the Android Application.

In a blockchain network, data is transferred with the help of transactions. For a transaction to occur, a PublicPrivate Key pair (linked to a Blockchain ID) is required. The Public and Private Keys are randomly generated strings of case sensitive letters and numbers which act as lock and key and hence provide authorization. Public Keys are widely distributed and used to identify the creator of a transaction. Private Keys are kept secret and used to create Digital Signatures. These signatures are then used to authenticate the creator and also verify data integrity.

Finding Nearest Drivers: The Prediction of the ANN model, as discussed in a previous section, helps to determine number of cab requests for a locality in advance and the corresponding number of cab drivers that need to be notified about the prediction. Selection of the best subset of all the available drivers can be performed by finding the ones nearest to the location of interest. KNN algorithm is used in the proposed system for the same. The algorithm takes as input a set of coordinates, a test data point and a value "K" (number of required co-ordinates). It gives as output $\mathrm{K}$ number of different coordinates from the set, which are closest to the test point. In case of the system described, input set of coordinates correspond to available cab locations and test data point represents the event's location. Prediction of the ANN model obtained earlier is the input " $\mathrm{K}$ " for the KNN model.

\section{KNN Algorithm:}

Step 1: Load the set of coordinates (Cab locations). Step 2: Load the value " $K$ " i.e. the required number of nearest cabs from the event location.

Step 3: For each data point in dataset:

- Calculate the distance between each point in the dataset and the test data point using Haversine Distance.

- Now, based on the distance value, sort the data points in ascending order.

- $\quad$ Choose top $\mathrm{K}$ data points from the sorted array.

Step 4: Provide chosen data points as output.

The "NearestNeighbor" class present in "sklearn. neighbors" library of Python Programming Language, is an unsupervised learner used for doing neighbor searches. It takes as input, the "number of Neighbors" and optional parameters like Radius, Algorithm, Metric. The "kneighbors" method of "NearestNeighbor" Class takes as input parameter, an array of features i.e set of locations of cabs, and returns a set of lengths i.e. the distance array and the corresponding indices of the nearest points .The distance and indices array are used to determine the nearest cab drivers to be notified. As location of event and drivers is in Latitude and Longitude, a distance metric called "Haversine Distance", which takes input in radians, is used. Haversine Distance is given by distance function:

\section{$\mathrm{d}=2 \arcsin \left(\operatorname{squt}\left(\sin ^{\wedge} 2\left(0.5^{*} \mathrm{dx}\right) \cos (\mathrm{x} 1) \cos (\mathrm{x} 2) \sin ^{\wedge} 2\left(0.5^{*} \mathrm{dy}\right)\right)\right)$}

The Latitudes and Longitudes of the locations obtained from the GPS are in degrees which is converted to radians. The conversion is performed by the use of an inbuilt function of Python i.e "math.radians". It is part of "math" library of Python Programming Language.

Integration of Blockchain with Database: As mentioned previously, a database system synchronizes data with the blockchain network. Synchronization is performed periodically to keep the database up-to-date. In a single synchronization cycle, data is fetched from the blockchain and then updated into the MongoDB database. Web3.js library is used to fetch the data from the blockchain cluster. Once the connection with the blockchain nodes, using specified IP addresses and port numbers is established, truffle-contract library helps to interact with the deployed contracts as explained in a previous section. To create the contract instance, a JSON file of the contract is used which was generated in the process of contract deployment. Using the contract instance, details of all the drivers are fetched from the blockchain.

The next step is storing these details into the database. The MongoDB JavaScript library has a function which takes URL of MongoDB database, Username and 
Password as input parameters to establish a connection with the database. Using the obtained connection instance, recently fetched details of all the cab drivers are updated in the database. The flow diagram of database synchronization is shown in Figure 1.

Figure 1: Database Synchronisation Flowchart

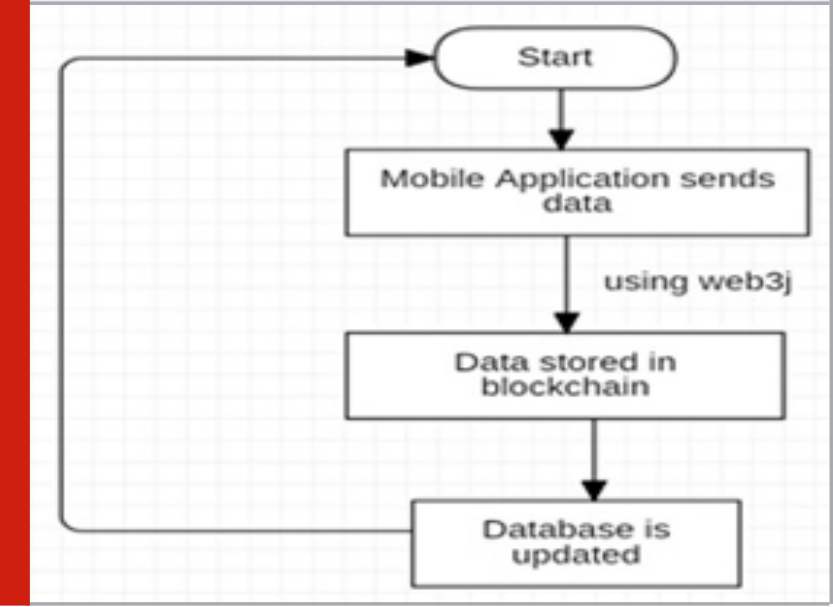

Figure 2: Flowchart of the integrated ML and KNN subsystem

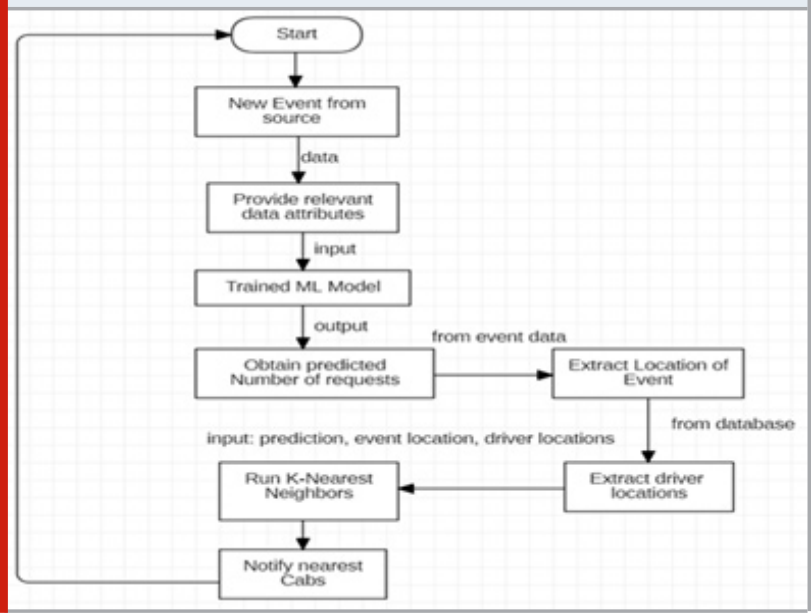

Integration of Machine Learning and $\mathrm{K}$-Nearest Neighbour: The ML and KNN scripts are implemented using Python Programming Language. Output of the ML script i.e the prediction of estimated number of bookings for an event is given as an input to KNN script along with the event location and the cab drivers' location. The integration of scripts is simple as they are written in the same programming language.

The Flowchart of the integrated ML and KNN subsystem is shown in Figure 2.

\section{CONCLUSION AND FUTURE SCOPE}

After integration of modules with the mentioned tools and libraries, a fully functional prototype system was developed. All the modules were found to be working in conjunction with each other. The conclusions drawn were as follows:

- It is feasible to use blockchain as a medium to transfer light data.

- The data transfer in blockchain took place in reasonable time frame for a realtime application.

- System is highly scalable because of distributed Blockchain network and features of Mongo DB.

- Accuracy of the ANN model was found to be acceptable.

\section{REFERENCES}

Grégoire Jeanmart(2019):Generate a Java Wrapper from your Smart Contract, https://kauri.io/article/844751323 17d4d6a84a2c42eb9348e4b/generate-a-java-wrapperfrom-your-smart-contract

Pauline Adam-Kalfon, Selsabila El Moutaouakil (2018). Blockchain, a catalyst for new approaches in insurance. pwc, 1, 8-9

Rajesh, RupalictChincholkar, Snehal (2018): A Study on Consumer Perception of Ola and Uber Taxi Services. 3. 25-31. 10.17010/ijcs/2018/v3/i5/138779.

Restuccia, Francesco \&td'oro, Salvatore \&tKanhere, SaliletMelodia, Tommaso\&t Das, Sajal (2018): Blockchain for the Internet of Things: Present and Future.

Zheng, Zibin\&Xie, Shaoandt Dai, Hong-Ningt Chen, Xiangping\&t Wang, Huaimin (2017) 2019: An Overview of Blockchain Technology: Architecture, Consensus, and Future Trends. 10.1109/BigDataCongress. 